\title{
THE SQUARES OF THE LAPLACIAN-DIRICHLET EIGENFUNCTIONS ARE GENERICALLY LINEARLY INDEPENDENT
}

\author{
YANNICK PRIVAT $^{1}$ AND MARIO Sigalottit ${ }^{1,2}$
}

\begin{abstract}
The paper deals with the genericity of domain-dependent spectral properties of the Laplacian-Dirichlet operator. In particular we prove that, generically, the squares of the eigenfunctions form a free family. We also show that the spectrum is generically non-resonant. The results are obtained by applying global perturbations of the domains and exploiting analytic perturbation properties. The work is motivated by two applications: an existence result for the problem of maximizing the rate of exponential decay of a damped membrane and an approximate controllability result for the bilinear Schrödinger equation.
\end{abstract}

Mathematics Subject Classification. 37C20, 47A55, 47A75, 49K20, 49K30, 93B05.

Received September 17, 2008. Revised March 11, 2009.

Published online July 2nd, 2009.

\section{INTRODUCTION}

Genericity is a measure of how much robust and frequent a property is. It enjoys, therefore, a deep-rooted success in control theory, where a generic behavior is, roughly speaking, the expected behavior of systems involving physical quantities whose value can only be approximated.

A paradigmatic example of generic properties in control theory is the controllability of a finite-dimensional linear system

$$
\dot{x}=A x+B u, \quad x \in \mathbb{R}^{n}, \quad u \in \mathbb{R}^{m} .
$$

It is well known, and the proof simply follows from the Kalman criterion, that for every choice of the positive integers $n$ and $m$ a generic linear system of type (1.1) is controllable. More precisely, the set of pairs $(A, B)$ for which (1.1) is controllable is open and dense in the product of the spaces of $n \times n$ and $n \times m$ matrices. (See, for instance, [26].)

When a control system involves partial differential equations, conditions guaranteeing its controllability, observability or stabilizability can often be stated in terms of the eigenvalues or eigenspaces of some linear operator (typically, the leading term of the evolution operator). In this paper we are mainly interested in conditions depending on the domain on which the control system of partial differential equations is defined. The genericity of some relevant conditions for control applications has already been considered and proved in the general field of partial differential equations (e.g., the simplicity of the eigenvalues of the Laplacian-Dirichlet

\footnotetext{
Keywords and phrases. Genericity, Laplacian-Dirichlet eigenfunctions, non-resonant spectrum, shape optimization, control.

${ }^{1}$ Institut Élie Cartan de Nancy, UMR 7502 Nancy-Université - INRIA - CNRS, B.P. 239, 54506 Vandœuvre-lès-Nancy Cedex, France.

${ }^{2}$ INRIA Nancy - Grand Est, France. Mario.sigalotti@inria.fr
} 
operator proved in [20,28] and applied in the control framework in [16]). Others, due to their technical nature, need to be tackled by specific arguments. This has led to the development of several tools for studying the genericity with respect to the domain of control-related properties of partial differential operators. Without seeking exhaustiveness, let us mention the works by Lions and Zuazua [17] and Ortega and Zuazua [23] on the Stokes system, those by Ortega and Zuazua [22,24] on the plate equation, the paper by Chitour et al. [8] on the heat and wave equations and the recent work by Beauchard et al. [6] on the Schrödinger equation.

The scope of this paper is to prove the genericity with respect to the domain of some properties of the Laplacian-Dirichlet operator issuing from control theory and optimization among which, in particular, the linear independence of the squared eigenfunctions.

In doing so we propose a technique that, we believe, has a wider range of applicability, going beyond the conditions studied here and adaptable to different operators. The difference between our approach and those usually adopted is that we focus less on local infinitesimal variations of the domain and more on global, longrange perturbations. In order to get genericity results from this kind of perturbations we have to rely on analytic-dependence properties for the eigenvalues and eigenfunctions of the Laplacian-Dirichlet operator with respect to analytic perturbations of the domain. (It should be stressed, however, that analytic perturbation theory applies to a much larger range of operators.) The idea of proving genericity through global perturbations is clearly not new, being intrinsically contained in analytic perturbation theory. Our work has actually been inspired by a paper by Hillairet and Judge [14], where the authors prove, using global perturbations, the generic simplicity of the eigenvalues of the Laplacian-Dirichlet operator on planar polygons with at least four vertices. The argument in [14], however, relies on the existence, in the class of interest, of domains having simple spectrum. The difficulty of extending the proof of [14] to show the generic linear independence of the squared eigenfunctions on smooth domains is that examples of smooth domains having the desired property are not handily available. One kind of domain on which the property can be easily checked is given by orthotopes. However, many results on spectral stability when non-smooth domains are approximated by smooth ones are known (see, in particular, the work by Arendt and Daners [3] where uniform stability of the eigenfunctions is studied) and imply the existence, for every $n \in \mathbb{N}$, of a smooth domain $\mathcal{R}_{n}$ whose first $n$ eigenfunctions have linearly independent squares. In order to propagate by global analytic perturbation the property satisfied by $\mathcal{R}_{n}$ one can use, for instance, exponential flows of vector fields (even a narrow family of vector fields is enough to generate a full orbit of domains, see [1]). One has, however, to take care of the possible crossing of the analytically depending eigenvalues. In order to do so, one should select analytic paths along which the first $n$ eigenvalues are simple. This problem is related to the Arnol'd conjecture (see $[4,10])$ and has been solved by Teytel in [27]. Teytel's result, recalled in Proposition 2.2, is crucial for the proposed perturbation technique (Thms. 2.3 and 2.4).

Let us conclude this introduction by describing the motivating applications of the properties that we consider. The generic linear independence of the squared eigenfunctions has been conjectured in dimension two by Hébrard and Henrot in [11], where the authors consider the problem of stabilizing with the largest possible decay rate a membrane fixed at its boundary using a damping acting on a portion of the membrane of fixed area. The existence and uniqueness of the solution for this problem can be deduced from the linear independence of the squared eigenfunctions of the Laplacian-Dirichlet operator on the domain filled by the membrane. (See Sect. 3 for more details.)

It should be noticed that whether such linear independence is not only generic but rather always true is still an open question. A negative result by Mahar and Willner [18] on the squared eigenfunctions of a Sturm-Liouville operator justify a cautious stance toward a conjecture saying that the linear independence should always hold true.

Linear independence of the squared eigenfunctions appears quite naturally also in the study of the controllability of the bilinear Schrödinger equation. In this context, indeed, non-resonance conditions on the spectrum 
of the uncontrolled Schrödinger operator are often required (see, for instance, [7]). Since the $k$ th eigenvalue $\lambda_{k}^{\varepsilon}$ of $-\Delta+\varepsilon V: H^{2}(\Omega) \cap H_{0}^{1}(\Omega) \rightarrow L^{2}(\Omega)$ is analytic with respect to $\varepsilon$ and satisfies

$$
\left.\frac{\mathrm{d}}{\mathrm{d} \varepsilon}\right|_{\varepsilon=0} \lambda_{k}^{\varepsilon}=\int_{\Omega} V(x) \phi_{k}(x)^{2} \mathrm{~d} x
$$

where $\left(\phi_{n}\right)_{n \in \mathbb{N}}$ is a complete system of eigenfunctions of $-\Delta$ (see [2]), then the linear independence of the family $\left(\phi_{n}^{2}\right)_{n \in \mathbb{N}}$ clearly plays a role in the study of the size of the family of potentials $V$ for which the spectrum has some prescribed property.

Another application discussed in Section 3 corresponds to the case where the uncontrolled Schrödinger operator is defined by a potential well, i.e., $V=0$ and $\Omega$ is free. We show in this case that, generically with respect to $\Omega$, no nontrivial linear combination with rational coefficients of the eigenvalues of $-\Delta$ annihilates. We deduce from this fact and the results in [7] a generic approximate controllability property for the Schrödinger equation.

Properties about the non-annihilation of linear combinations of eigenvalues play a role also in other domains. Let us mention, for instance, the recent work by Zuazua on switching systems in infinite dimension [29], where the condition that the sums of two different pairs of eigenvalues of the Laplacian-Dirichlet operator are different is used to prove null-controllability of the heat equation using switching controls.

The paper is organized as follows: in Section 2 we introduce some definitions and notations and we prove the main abstract results of the paper (Thms. 2.3 and 2.4). We conclude the section by deducing from the abstract results some specific generic conditions; in particular, we obtain the generic linear independence of the squared eigenfunctions of the Laplacian-Dirichlet operator. In Section 3 we propose two applications of these generic properties to the stabilization of vibrating membranes and to the controllability of the Schrödinger equation.

\section{Generic properties by global perturbations}

\subsection{Notations and abstract genericity result}

Throughout the paper, $d$ denotes an integer larger than or equal to two and $\mathbb{N}$ the set of positive integer numbers, while $\mathbb{N}_{0}=\{0\} \cup \mathbb{N}$.

Given a Lipschitz domain $\Omega \subset \mathbb{R}^{d}$, we denote by $\left(\lambda_{n}^{\Omega}\right)_{n \in \mathbb{N}}$ the nondecreasing sequence of eigenvalues of the Laplacian-Dirichlet operator

$$
-\Delta: H^{2}(\Omega) \cap H_{0}^{1}(\Omega) \rightarrow L^{2}(\Omega)
$$

counted according to their multiplicity. As it is well known, it is always possible to choose an orthonormal basis of $L^{2}(\Omega)$ made of eigenfunctions of the Laplacian-Dirichlet operator. In the sequel any such choice will be denoted by $\left(\phi_{n}^{\Omega}\right)_{n \in \mathbb{N}}$ with $\phi_{n}^{\Omega}$ corresponding to the eigenvalue $\lambda_{n}^{\Omega}$. We will identify $\phi_{n}^{\Omega}$ with its extension to zero outside $\Omega$.

We define the class of domains $\Sigma_{m}$ as the set of open subsets of $\mathbb{R}^{d}$ with $\mathcal{C}^{m}$ boundary. By $D_{m}$ we denote the subset of $\Sigma_{m}$ of $\mathcal{C}^{m}$ topological balls, i.e., those open subsets $\Omega$ of $\mathbb{R}^{d}$ such that there exists a $\mathcal{C}^{m}$-diffeomorphism of $\mathbb{R}^{d}$ transforming the unit ball in $\Omega$. Similarly, we define $D_{0,1}$ as the orbit of the unit ball by bi-Lipschitz homeomorphisms of $\mathbb{R}^{d}$.

It is well known that $\Sigma_{m}$ and $D_{m}$, endowed with the $\mathcal{C}^{m}$ topology inherited from that of $\mathcal{C}^{m}$-diffeomorphisms, are complete metric spaces (see [19]). In particular, they are Baire spaces.

Let us recall that, given a Baire space $X$, a residual set (i.e. the intersection of countably many open and dense subsets) is dense in $X$. A boolean function $\mathcal{P}: X \rightarrow\{0,1\}$ is said to be generic in $X$ if there exists a residual set $Y$ such that every $x$ in $Y$ satisfies property $\mathcal{P}$, that is, $\mathcal{P}(x)=1$.

A sequence of open domains $\left(\Omega_{n}\right)_{n \in \mathbb{N}}$ is said to compactly converge to a domain $\Omega$ if for every compact set $K \subseteq \Omega \cup \bar{\Omega}^{c}$, there exists $n_{K} \in \mathbb{N}$ such that for all $n \geq n_{K}, K \subseteq \Omega_{n} \cup{\overline{\Omega_{n}}}^{c}$.

In the sequel of the paper, we make use several times of the following result, whose proof can be found in [3], Theorem 7.3. 
Proposition 2.1. Let $n \in \mathbb{N}$ and fix a Lipschitz domain $\Omega \subset \mathbb{R}^{d}$ such that $\lambda_{1}^{\Omega}, \ldots, \lambda_{n}^{\Omega}$ are simple. Let $\Omega_{k}$ be a sequence of Lipschitz domains compactly converging to $\Omega$ and such that $\cup_{k \in \mathbb{N}} \Omega_{k}$ is bounded. Then $\lambda_{j}^{\Omega_{k}} \rightarrow \lambda_{j}^{\Omega}$ and, therefore, $\lambda_{j}^{\Omega_{k}}$ is simple for every $j=1, \ldots, n$, for $k$ large enough. Moreover, up to a sign in the choice of $\phi_{j}^{\Omega_{k}}, \phi_{j}^{\Omega_{k}} \rightarrow \phi_{j}^{\Omega}$ in $L^{\infty}\left(\mathbb{R}^{d}\right)$, as $k$ goes to infinity, for $j=1, \ldots, n$.

Another result playing a crucial role in our argument is the following theorem, due to Teytel (see [27], Thm. 6.4).

Proposition 2.2. Let $m>2$ and $\Omega_{0}, \Omega_{1}$ be two simply connected domains of $\mathbb{R}^{d}$ that are $\mathcal{C}^{m}$-differentiably isotopic to the unit d-dimensional ball. Then there exists an analytic curve $[0,1] \ni t \mapsto Q_{t}$ of $C^{m}$-diffeomorphisms such that $Q_{0}$ is equal to the identity, $Q_{1}\left(\Omega_{0}\right)=\Omega_{1}$ and every domain $\Omega_{t}=Q_{t}\left(\Omega_{0}\right)$ has simple spectrum for $t$ in the open interval $(0,1)$.

Teytel deduces the proposition stated above from a more general result, namely [27], Theorem B, that guarantees the existence of an analytic path of simple-spectrum operators among any elements of a family of operators satisfying a strong Arnold hypothesis on theirs eigenvectors (see also [4,10]). For this reason we expect that our method could be adapted to other situations.

We are ready to prove the following theorem on generic properties among topological balls.

Theorem 2.3. Let $F_{n}: \mathbb{R}^{n(n+1)} \longrightarrow \mathbb{R}, n \in \mathbb{N}$, be a sequence of analytic functions. For every $n \in \mathbb{N}$, we say that a Lipschitz domain $\Omega$ satisfies property $\mathcal{P}_{n}$ if $\lambda_{1}^{\Omega}, \ldots, \lambda_{n}^{\Omega}$ are simple and if there exist $n$ points $x_{1}, \ldots, x_{n}$ in $\Omega$ and a choice $\phi_{1}^{\Omega}, \ldots, \phi_{n}^{\Omega}$ of the first $n$ eigenfunctions of the Laplacian-Dirichlet operator on $\Omega$ such that

$$
F_{n}\left(\phi_{1}^{\Omega}\left(x_{1}\right), \ldots, \phi_{n}^{\Omega}\left(x_{1}\right), \ldots, \phi_{1}^{\Omega}\left(x_{n}\right), \ldots, \phi_{n}^{\Omega}\left(x_{n}\right), \lambda_{1}^{\Omega}, \ldots, \lambda_{n}^{\Omega}\right) \neq 0
$$

Assume that, for every $n \in \mathbb{N}$, there exists $\mathcal{R}_{n} \in D_{0,1}$ satisfying property $\mathcal{P}_{n}$. Then, for every $m \in \mathbb{N} \cup\{+\infty\}$, a generic $\Omega \in D_{m}$ satisfies $\mathcal{P}_{n}$ for every $n \in \mathbb{N}$.

Proof. Fix $m \in \mathbb{N} \cup\{+\infty\}$. Define, for every $n \in \mathbb{N}$, the set of domains

$$
\mathcal{A}_{n}=\left\{\Omega \in D_{m} \mid \Omega \text { satisfies } \mathcal{P}_{n}\right\}
$$

We shall fix $n \in \mathbb{N}$ and prove that each $\mathcal{A}_{n}$ is open and dense in $D_{m}$.

Let us first prove that $\mathcal{A}_{n}$ is open. Fix $\Omega \in \mathcal{A}_{n}$, a choice of eigenfunctions $\phi_{1}^{\Omega}, \ldots, \phi_{n}^{\Omega}$ and $n$ points $x_{1}, \ldots, x_{n} \in \Omega$ such that $(2.1)$ holds true. Suppose by contradiction that there exists a sequence $\left(\Omega_{k}\right)_{k \in \mathbb{N}}$ in $D_{m} \backslash \mathcal{A}_{n}$ that converges to $\Omega$. Notice that the convergence in $D_{m}$ implies compact convergence in the sense recalled above. Proposition 2.1 thus implies that, for a choice of $\phi_{j}^{\Omega_{k}}, j=1, \ldots, n$, one has

$$
\lim _{k \rightarrow+\infty} F_{n}\left(\phi_{1}^{\Omega_{k}}\left(x_{1}\right), \ldots, \phi_{n}^{\Omega_{k}}\left(x_{n}\right), \lambda_{1}^{\Omega_{k}}, \ldots, \lambda_{n}^{\Omega_{k}}\right)=F_{n}\left(\phi_{1}^{\Omega}\left(x_{1}\right), \ldots, \phi_{n}^{\Omega}\left(x_{n}\right), \lambda_{1}^{\Omega}, \ldots, \lambda_{n}^{\Omega}\right) \neq 0
$$

This contradicts the assumption that $\Omega_{k} \notin \mathcal{A}_{n}$ for every $k \in \mathbb{N}$.

We prove now the density of $\mathcal{A}_{n}$. Notice that, without loss of generality, $m>2$. Fix $\Omega \in D_{m}$. Let $\mathcal{R}_{n}$ be as in the statement of the theorem, that is, $\mathcal{R}_{n} \in D_{0,1}$ and satisfies property $\mathcal{P}_{n}$. Notice that $\mathcal{R}_{n}$ can be approximated by a sequence of domains in $D_{m}$ in the sense of the compact convergence. Therefore, by applying the same argument as above, we deduce that there exists $\widetilde{\mathcal{R}}_{n} \in D_{m}$ satisfying $\mathcal{P}_{n}$. Choose $\phi_{j}^{\widetilde{\mathcal{R}}_{n}}, j=1, \ldots, n$, and $x_{1}, \ldots, x_{n} \in \widetilde{\mathcal{R}}_{n}$ such that

$$
F_{n}\left(\phi_{1}^{\widetilde{\mathcal{R}}_{n}}\left(x_{1}\right), \ldots, \phi_{n}^{\widetilde{\mathcal{R}}_{n}}\left(x_{n}\right), \lambda_{1}^{\widetilde{\mathcal{R}}_{n}}, \ldots, \lambda_{n}^{\widetilde{\mathcal{R}}_{n}}\right) \neq 0
$$

We now apply Proposition 2.2 with $\Omega_{0}=\widetilde{\mathcal{R}}_{n}$ and $\Omega_{1}=\Omega$. We deduce that, for $m>2$, there exists an analytic curve $[0,1] \ni t \mapsto Q_{t}$ of $C^{m}$-diffeomorphisms such that $Q_{0}$ is equal to the identity, $Q_{1}\left(\widetilde{\mathcal{R}}_{n}\right)=\Omega$ 
and every domain $\Omega_{t}=Q_{t}\left(\widetilde{\mathcal{R}}_{n}\right) \in D_{m}$ has simple spectrum for $t$ in the open interval $(0,1)$. Due to standard analytic perturbation theory (see [15]), $\lambda_{k}^{\Omega_{t}}$ are analytic functions of $t$ and there exists a choice of $\phi_{j}^{\Omega_{t}}$, $j=1, \ldots, n, t \in[0,1]$, such that $\phi_{j}^{\Omega_{t}} \circ Q_{t}$ varies analytically with respect to $t$ in $\mathcal{C}^{m}\left(\widetilde{\mathcal{R}}_{n}\right)$. In particular,

$$
t \mapsto F_{n}\left(\phi_{1}^{\Omega_{t}}\left(Q_{t}\left(x_{1}\right)\right), \ldots, \phi_{n}^{\Omega_{t}}\left(Q_{t}\left(x_{n}\right)\right), \lambda_{1}^{\Omega_{t}}, \ldots, \lambda_{n}^{\Omega_{t}}\right)
$$

is an analytic real-valued function. Since its value at $t=0$ is different from zero, then it annihilates only for finitely many $t \in[0,1]$.

Hence, as required, $\Omega$ can be approximated arbitrarily well in $D_{m}$ by an element of $\mathcal{A}_{n}$.

Let us turn our attention to domains that are not necessarily topological balls. The point where this topological assumption plays a fundamental role in the proof above is in the application of Proposition 2.2 guaranteeing that smooth topological balls can be deformed one into another through an analytic path preserving the strict order of the eigenvalues. In order to extend the genericity result to domains in $\Sigma_{m}$, we impose a more restrictive assumption on the family of analytic test functions $F_{n}$. Roughly speaking, we ask it to be invariant by reordering of the eigenvalues.

Theorem 2.4. Let $F_{n}: \mathbb{R}^{n(n+1)} \longrightarrow \mathbb{R}, n \in \mathbb{N}$, be a sequence of analytic functions. For every $n \in \mathbb{N}$ define $J_{n}$ as the subset of $\mathbb{N}^{n}$ made of all $n$-uples of pairwise distinct positive integer numbers. Given $j=\left(j_{1}, \ldots, j_{n}\right)$ in $J_{n}$, we say that a Lipschitz domain $\Omega$ satisfies property $\hat{\mathcal{P}}_{j}$ if $\lambda_{j_{1}}^{\Omega}, \ldots, \lambda_{j_{n}}^{\Omega}$ are simple and if there exist $n$ points $x_{1}, \ldots, x_{n}$ in $\Omega$ and a choice $\phi_{1}^{\Omega}, \ldots, \phi_{\|j\|_{\infty}}^{\Omega}$ of the first $\|j\|_{\infty}$ eigenfunctions of the Laplacian-Dirichlet operator on $\Omega$ such that

$$
F_{n}\left(\phi_{j_{1}}^{\Omega}\left(x_{1}\right), \ldots, \phi_{j_{n}}^{\Omega}\left(x_{1}\right), \ldots, \phi_{j_{1}}^{\Omega}\left(x_{n}\right), \ldots, \phi_{j_{n}}^{\Omega}\left(x_{n}\right), \lambda_{j_{1}}^{\Omega}, \ldots, \lambda_{j_{n}}^{\Omega}\right) \neq 0 .
$$

Assume that, for every $n \in \mathbb{N}$ and $j \in J_{n}$, there exists $\hat{\mathcal{R}}_{j} \in D_{0,1}$ satisfying property $\hat{\mathcal{P}}_{j}$. Then, for every $m \in \mathbb{N} \cup\{+\infty\}$, a generic $\Omega \in \Sigma_{m}$ satisfies $\hat{\mathcal{P}}_{j}$ for every $j \in \cup_{n \in \mathbb{N}} J_{n}$.

Proof. Fix $m \in \mathbb{N} \cup\{+\infty\}$. Notice that, by ordering $J_{\infty}:=\cup_{n \in \mathbb{N}} J_{n}$, the hypotheses of the theorem are easily transformed in a special case of those of Theorem 2.3. Therefore, a generic $\hat{\Omega} \in D_{m}$ satisfies $\hat{\mathcal{P}}_{j}$ for every $j \in J_{\infty}$. Fix one such $\hat{\Omega}$ and notice that, in particular, the spectrum $\left(\lambda_{n}^{\hat{\Omega}}\right)_{n \in \mathbb{N}}$ is simple.

Define, for every $j \in J_{\infty}$, the set

$$
\hat{\mathcal{A}}_{j}=\left\{\Omega \in \Sigma_{m} \mid \Omega \text { satisfies } \hat{\mathcal{P}}_{j}\right\}
$$

The openness of $\hat{\mathcal{A}}_{j}$ in $\Sigma_{m}$ can be proved following exactly the same argument used in the proof of Theorem 2.3 to show that each $A_{n}$ is open in $D_{m}$.

We are left to prove that $\hat{\mathcal{A}}_{j}$ is dense in $\Sigma_{m}$. Take $\Omega \in \Sigma_{m}$. Let $B$ be an open ball of $\mathbb{R}^{d}$ containing $\Omega$. By eventually shrinking $B$, we can assume that $\partial B \cap \partial \Omega$ contains at least one point $p$. Up to a change of coordinates, we can assume that $B$ is centered at the origin and $p=(0, \ldots, 0,1)$.

Consider a smooth vector field on $\mathbb{R}^{d}$ satisfying

$$
V\left(x_{1}, \ldots, x_{d}\right)=\left\{\begin{array}{c}
\left(\begin{array}{c}
x_{1} x_{d} \\
\vdots \\
x_{d-1} x_{d} \\
x_{d}^{2}-\frac{x_{1}^{2}+\ldots+x_{d}^{2}+1}{2}
\end{array}\right) \\
\text { if } x_{1}^{2}+\ldots+x_{d}^{2}<\rho \\
\text { if } x_{1}^{2}+\ldots+x_{d}^{2}>\rho+1
\end{array}\right.
$$

for some $\rho>1$. The behavior of $V$ in a neighborhood of the unit ball is represented in Figure 1. Notice that $V$ is complete, since it vanishes outside a compact set. 


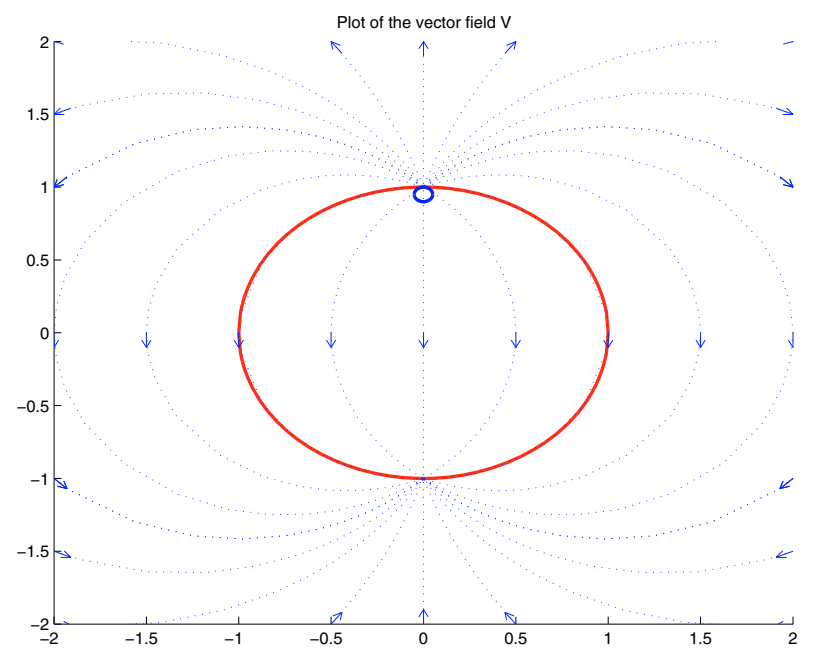

Figure 1. Phase portrait of the vector field $V$.

By construction $V$ is everywhere tangent to $\partial B$. The ball $B$ is therefore invariant for the flow of $V$. Notice that the points $p$ and $-p$ are the only zeros of $V$ in $\bar{B}$ and that $x_{d}$ is strictly decreasing along all trajectories of $V$ staying in $B$. Therefore, $p$ is a repulsive equilibrium for $V$ restricted to $\bar{B}$ and $-p$ an attractive one.

Notice that, since $\Omega \in \Sigma_{m}$, then its boundary has finitely many components and therefore there exists a ball $B^{\prime}$ contained in $\Omega$ such that $p \in \partial B^{\prime}$. Notice, moreover, that the differential of $V$ at $\pm p$ is $\pm \mathrm{Id}$. Then, for every $x \in B$, $\mathrm{e}^{-t V}(x)$ belongs to $B^{\prime}$ for every $t$ larger than some $t_{x} \in \mathbb{R}$. We deduce that $\mathrm{e}^{t V}\left(B^{\prime}\right)$ compactly converges to $B$ as $t$ tends to infinity. Since $B^{\prime} \subset \Omega$, then $\mathrm{e}^{t V}(\Omega)$ compactly converges to $B$ as well as $t \rightarrow+\infty$.

Consider an analytic path $t \mapsto Q_{t}$ of $\mathcal{C}^{m}$-diffeomorphisms of $\mathbb{R}^{d}$ such that $Q_{0}=\operatorname{Id}$ and $Q_{1}(B)=\hat{\Omega}$, whose existence can be deduced from Proposition 2.2. Then

$$
\Omega_{t}=Q_{\frac{2 \arctan t}{\pi}} \circ \mathrm{e}^{t V}(\Omega) \text { compactly converges to } \hat{\Omega} \text { as } t \rightarrow+\infty
$$

Moreover, $t \mapsto \Omega_{t}$ is an analytic path in $\Sigma_{m}$. Hence, there exist $n$ analytic functions $\Lambda_{1}, \ldots, \Lambda_{n}:[0,+\infty) \rightarrow \mathbb{R}$ such that $\Lambda_{k}(0)=\lambda_{j_{k}}^{\Omega}$ and $\Lambda_{k}(t)$ is in the spectrum of the Laplacian-Dirichlet operator on $\Omega_{t}$, for $k=1, \ldots, n$. Moreover, there exist $n$ functions $\Phi_{1}, \ldots, \Phi_{n}$ defined on $[0,+\infty)$ such that $\Phi_{k}(t) \in C^{\infty}\left(\Omega_{t}\right)$ is an eigenfunction corresponding to $\Lambda_{k}(t)$, for $k=1, \ldots, n$ and $t \in[0,+\infty)$, and $t \mapsto \Phi_{k}(t) \circ Q_{\frac{2 \arctan t}{\pi}}^{\pi} \circ \mathrm{e}^{t V}$ varies analytically with respect to $t$ in $\mathcal{C}^{m}(\Omega)$. Let us write $\Phi_{k}(t, x)$ for the evaluation of $\Phi_{k}(t)$ at a point $x \in \Omega_{t}$.

We claim that each $\Lambda_{k}(t)$ converges, as $t \rightarrow+\infty$, to an eigenvalue of the Laplacian-Dirichlet operator on $\hat{\Omega}$. Indeed, the compact convergence of $\Omega_{t}$ to $\hat{\Omega}$ implies the strong convergence of the corresponding resolvent operators (see, for instance, [3]). This, in turns, guarantees that $\left|\lambda_{i}^{\Omega_{t}}-\lambda_{i}^{\hat{\Omega}}\right| \leq \varepsilon(t)$ with $\varepsilon(t)$ independent of $i$ and converging to zero as $t \rightarrow+\infty$. Hence, there exists $M \in \mathbb{N}$ such that for every $t \geq 0$ and every $k=1, \ldots, n$ there exists $i \leq M$ such that $\Lambda_{k}(t)=\lambda_{i}^{\Omega_{t}}$. In particular, according to Proposition 2.1, there exist $\left(i_{1}, \ldots, i_{n}\right) \in J_{n}$ such that $\Lambda_{k}(t) \rightarrow \lambda_{i_{k}}^{\hat{\Omega}}$ as $t \rightarrow+\infty$ for $k=1, \ldots, n$.

Moreover, up to a sign in the choice of $\phi_{i_{k}}^{\hat{\Omega}}$, we can assume that $\Phi_{k}(t) \rightarrow \phi_{i_{k}}^{\hat{\Omega}}$ in $L^{\infty}\left(\mathbb{R}^{d}\right)$, as $t \rightarrow \infty$, for $k=1, \ldots, n$. The choice of $\hat{\Omega}$ guarantees that it contains $x_{1}, \ldots, x_{n}$ such that

$$
F_{n}\left(\phi_{i_{1}}^{\hat{\Omega}}\left(x_{1}\right), \ldots, \phi_{i_{n}}^{\hat{\Omega}}\left(x_{n}\right), \lambda_{i_{1}}^{\hat{\Omega}}, \ldots, \lambda_{i_{n}}^{\hat{\Omega}}\right) \neq 0 .
$$


For $\tau$ large enough $x_{1}, \ldots, x_{n}$ belong to $\Omega_{\tau}$ and

$$
F_{n}\left(\Phi_{1}\left(\tau, x_{1}\right), \ldots, \Phi_{n}\left(\tau, x_{n}\right), \Lambda_{1}(\tau), \ldots, \Lambda_{n}(\tau)\right) \neq 0 .
$$

Fix one such $\tau$ and define

$$
\bar{x}_{k}=\mathrm{e}^{-\tau V} \circ Q_{\frac{2 \arctan \tau}{\pi}}^{-1}\left(x_{k}\right)
$$

for $k=1, \ldots, n$. Therefore,

$$
t \mapsto F_{n}\left(\Phi_{1}\left(t, Q_{\frac{2 \arctan t}{\pi}} \circ \mathrm{e}^{t V}\left(\bar{x}_{1}\right)\right), \ldots, \Phi_{n}\left(t, Q_{\frac{2 \arctan t}{\pi}} \circ \mathrm{e}^{t V}\left(\bar{x}_{n}\right)\right), \Lambda_{1}(t), \ldots, \Lambda_{n}(t)\right)
$$

is an analytic real-valued function which has a nonzero value for $t=\tau$. Hence, there exists $t$ arbitrarily small such that

$$
F_{n}\left(\Phi_{1}\left(t, Q_{\frac{2 \arctan t}{\pi}}^{\pi} \circ \mathrm{e}^{t V}\left(\bar{x}_{1}\right)\right), \ldots, \Phi_{n}\left(t, Q_{\frac{2 \arctan t}{\pi}} \circ \mathrm{e}^{t V}\left(\bar{x}_{n}\right)\right), \Lambda_{1}(t), \ldots, \Lambda_{n}(t)\right) \neq 0 .
$$

Since, for $t$ small enough, $\Lambda_{k}(t)=\lambda_{j_{k}}^{\Omega_{t}}$, we deduce that $\Omega$ can be approximated arbitrarily well in $\Sigma_{m}$ by an element of $\hat{\mathcal{A}}_{j}$.

\subsection{Consequences of the abstract results}

In this section, we present two corollaries of Theorem 2.4 showing that (i) the squares of the LaplacianDirichlet eigenfunctions are generically linearly independent and (ii) the Laplacian-Dirichlet spectrum is generically non-resonant.

Recall that a finite or infinite sequence of real numbers is said to be non-resonant if every nontrivial rational linear combination of finitely many of its elements is different from zero.

In order to verify that the squares of the Laplacian-Dirichlet eigenfunctions on a suitably chosen $d$-orthotope are linearly independent, we prove the following technical result.

Lemma 2.5. Let $\varphi$ belong to $\mathcal{C}^{\infty}([0,+\infty), \mathbb{R}), N$ be a positive integer and $\left(a_{1}, \ldots, a_{N}\right)$ be a sequence of pairwise distinct positive real numbers. Assume that there exist $l_{0} \in \mathbb{N}_{0}$ and $l_{1} \in \mathbb{N}$ such that $\varphi^{\left(l_{0}+p l_{1}\right)}(0) \neq 0$ for every $p=0, \ldots, N-1$. Then, the functions $\varphi\left(a_{1} \cdot\right), \ldots, \varphi\left(a_{N} \cdot\right)$ are linearly independent on every right-neighborhood of zero.

Proof. We are interested in finding all the $N$-tuples $\left(\gamma_{1}, \ldots, \gamma_{N}\right) \in \mathbb{R}^{N}$ such that $\sum_{k=1}^{N} \gamma_{k} \varphi\left(a_{k} \cdot\right)=0$ in a rightneighborhood of zero. Differentiating this relation $l_{0}+p l_{1}$ times yields the relation $\sum_{k=1}^{N} \gamma_{k} a_{k}^{l_{0}+p l_{1}} \varphi^{\left(l_{0}+p l_{1}\right)}\left(a_{k} \cdot\right)=$ 0 . Evaluating such relation at zero for $p=0, \ldots, N-1$, we obtain a system of $N$ linear equations in the $N$ variables $\gamma_{1}, \ldots, \gamma_{N}$. Since $\left(a_{j}^{l_{1}(i-1)}\right)_{1 \leq i, j \leq N}$ is a Vandermonde matrix, the determinant $\delta_{N}$ of the $N \times N$ matrix underlying such a system writes

$$
\begin{aligned}
\delta_{N} & =\operatorname{det}\left(\left(a_{j}^{l_{1}(i-1)}\right)_{1 \leq i, j \leq N}\right) \prod_{k=1}^{N} a_{k}^{l_{0}} \varphi^{\left(l_{0}+k l_{1}\right)}(0) \\
& =\prod_{1 \leq i<j \leq N}\left(a_{j}^{l_{0}}-a_{i}^{l_{0}}\right) \prod_{k=1}^{N} a_{k}^{l_{0}} \varphi^{\left(l_{0}+k l_{1}\right)}(0) \neq 0 .
\end{aligned}
$$

This concludes the proof of the lemma.

Proposition 2.6. Let $\left(\mu_{1}, \ldots, \mu_{d}\right)$ be a non-resonant sequence of positive real numbers and $\mathcal{R}$ be the $d$-orthotope $\prod_{i=1}^{d}\left(0, \mu_{i} \pi\right)$. Then, the Laplacian-Dirichlet eigenvalues of $\mathcal{R}$ are simple and the squares of the LaplacianDirichlet eigenfunctions are linearly independent. 
Proof. Let us prove the lemma by induction on $d \geq 1$.

If $d=1$, then $\mu_{1}$ is any positive real number and the squared eigenfunctions of the Laplacian-Dirichlet operator on $\mathcal{R}$ are $\left(\sin ^{2}\left(k / \mu_{1}\right)\right)_{k \in \mathbb{N}}$. The proposition follows then from Lemma 2.5, applied to $\varphi(x)=\sin ^{2}(x)$, $l_{0}=1, l_{1}=2$, and $a_{k}=k / \mu_{1}$.

Let now $d$ be larger than one. For $K=\left(k_{1}, \ldots, k_{d}\right) \in \mathbb{N}^{d}$, we write $K^{\prime}=\left(k_{1}, \ldots, k_{d-1}\right), \bar{K}=k_{d}$ and we denote by $f_{K}$ the (un-normalized) Laplacian-Dirichlet eigenfunction

$$
f_{K}\left(x_{1}, \ldots, x_{d}\right)=\prod_{i=1}^{d} \sin \left(\frac{k_{i} x_{i}}{\mu_{i}}\right) .
$$

Clearly, $f_{K}\left(x_{1}, \ldots, x_{d}\right)=f_{K^{\prime}}\left(x_{1}, \ldots, x_{d-1}\right) \sin \left(\bar{K} x_{d} / \mu_{d}\right)$. Fix $I \subset \mathbb{N}^{d}$ finite and $\left\{\gamma_{K} \mid K \in I\right\} \subset \mathbb{R}$ such that $\sum_{K \in I} \gamma_{K} f_{K}^{2} \equiv 0$ on $\mathcal{R}$. Let $\bar{I}=\{\bar{K} \mid K \in I\}$. Then for every $\left(x_{1}, \ldots, x_{d-1}\right) \in \prod_{i=1}^{d-1}\left(0, \mu_{i} \pi\right)$ and every $x_{d} \in\left(0, \mu_{d} \pi\right)$ we have

$$
\sum_{k \in \bar{I}}\left(\sum_{K \in I, \bar{K}=k} \gamma_{K} f_{K^{\prime}}\left(x_{1}, \ldots, x_{d-1}\right)^{2}\right) \sin ^{2}\left(\frac{k x_{d}}{\mu_{d}}\right)=0 .
$$

Therefore, applying again Lemma 2.5 to $\varphi(x)=\sin ^{2}(x)$, we deduce that, for every $k \in \bar{I}$,

$$
\sum_{K \in I, \bar{K}=k} \gamma_{K} f_{K^{\prime}}^{2} \equiv 0 \quad \text { on } \prod_{i=1}^{d-1}\left(0, \mu_{i} \pi\right)
$$

The induction hypothesis implies that $\gamma_{K}$ is equal to zero for every $K$ such that $\bar{K}=k$. Since $k$ is arbitrary in $\bar{I}$, the proposition is proved.

We can now state the first corollary of Theorem 2.4.

Corollary 2.7. Let $m \in \mathbb{N} \cup\{\infty\}$. Generically with respect to $\Omega \in \Sigma_{m}$, the squares of the Laplacian-Dirichlet eigenfunctions are linearly independent when restricted to any measurable subset of $\Omega$ of positive measure.

Proof. First notice that $n$ functions $\varphi_{1}, \ldots, \varphi_{n}$ defined on a domain $\Omega$ are linearly independent if and only if there exist $n$ points $x_{1}, \ldots, x_{n}$ in $\Omega$ such that

$$
\operatorname{det}\left(\begin{array}{ccc}
\varphi_{1}\left(x_{1}\right) & \ldots & \varphi_{n}\left(x_{1}\right) \\
\vdots & & \vdots \\
\varphi_{1}\left(x_{n}\right) & \ldots & \varphi_{n}\left(x_{n}\right)
\end{array}\right) \neq 0 .
$$

Apply Theorem 2.4 with

$$
F_{n}\left(y_{1}, \ldots, y_{n(n+1)}\right)=\operatorname{det}\left(\begin{array}{ccc}
y_{1} & \ldots & y_{n} \\
\vdots & & \vdots \\
y_{n^{2}-n+1} & \ldots & y_{n^{2}}
\end{array}\right),
$$

for $\left(y_{1}, \ldots, y_{n(n+1)}\right) \in \mathbb{R}^{n(n+1)}$, and $\hat{\mathcal{R}}_{j}=\mathcal{R}$ for every $j \in J_{n}$, where $\mathcal{R}$ is the $d$-orthotope introduced in the statement of Proposition 2.6.

Then for a generic $\Omega \in \Sigma_{m}$ the squares of the Laplacian-Dirichlet eigenfunctions are linearly independent on $\Omega$. Assume that there exists a measurable subset $\mathcal{O} \subset \Omega$ of positive measure and $K$ constants $\gamma_{1}, \ldots, \gamma_{K}$ such that $\sum_{k=1}^{K} \gamma_{k} \phi_{k}^{\Omega}(x)^{2}=0$ on $\mathcal{O}$. Recall now that the hypo-analyticity of the Laplacian operator implies that each eigenfunction is analytic inside $\Omega$. Hence $\gamma_{1}=\ldots=\gamma_{K}=0$.

Corollary 2.7 can be used to get generic spectral properties as in [29], Section 6.3.

Another consequence of Theorem 2.4 is the following corollary. 
Corollary 2.8. Fix $m \in \mathbb{N} \cup\{\infty\}, k \in \mathbb{N}$ and $q=\left(q_{1}, \ldots, q_{k}\right) \in \mathbb{R}^{k} \backslash\{0\}$. Then, for a generic $\Omega \in \Sigma_{m}$ one has

$$
\sum_{l=1}^{k} q_{l} \lambda_{l}^{\Omega} \neq 0
$$

In particular, a generic $\Omega \in \Sigma_{m}$ has non-resonant spectrum.

Proof. Let $\mathcal{R}$ be a $d$-orthotope defined as in the statement of Proposition 2.6.

We denote by $\Gamma$ the subset of $\partial \mathcal{R}$ defined by

$$
\Gamma=\left\{\left(x_{1}, \ldots, x_{d}\right) \in \partial \mathcal{R} \mid x_{d}=\mu_{d} \pi\right\} .
$$

Consider a perturbation $\mathcal{R}^{t}:=(\operatorname{Id}+t V)(\mathcal{R})$ of the domain $\mathcal{R}$, with $t$ small and $V$ a smooth vector field whose support is compact and does not intersect $\partial \mathcal{R} \backslash \Gamma$. Then, it is well known (see, e.g. [13,21,25]) that, since the Laplacian-Dirichlet eigenvalues of $\mathcal{R}$ are simple, the shape derivative of $\lambda_{l}^{\mathcal{R}}$ along $V$ is defined as

$$
\left\langle\mathrm{d} \lambda_{l}^{\mathcal{R}}, V\right\rangle=\left.\frac{\mathrm{d}}{\mathrm{d} t} \lambda_{l}^{\mathcal{R}^{t}}\right|_{t=0}=-\int_{\Gamma}\left(\frac{\partial \phi_{l}^{\mathcal{R}}}{\partial \nu}\right)^{2}(V \cdot \nu) \mathrm{d} \sigma
$$

where $\nu$ denotes the outward normal to $\mathcal{R}$ and $d \sigma$ the $(d-1)$-dimensional surface element. By hypothesis $\nu=(0, \ldots, 0,1)$ on $\Gamma$, so that $V \cdot \nu$ is equal to $v_{d}$, the $d$ th component of $V$. Notice, moreover, that

$$
\frac{\partial \phi_{l}^{\mathcal{R}}}{\partial \nu}=c_{l} f_{K_{l}}
$$

for some nonzero constant $c_{l} \in \mathbb{R}$ (defined up to sign) and some $K_{l} \in \mathbb{N}^{d-1}$, where $f_{K_{l}}$ is defined as in the proof of Proposition 2.6.

Let $q=\left(q_{1}, \ldots, q_{k}\right) \in \mathbb{R}^{k} \backslash\{0\}, j \in J_{k}$ and introduce $G_{j}: \Omega \mapsto \sum_{l=1}^{k} q_{l} \lambda_{j_{l}}^{\Omega}$. Differentiating $G_{j}$ at $\Omega=\mathcal{R}$ along a vector field $V$ chosen as above yields,

$$
\left\langle\mathrm{d} G_{j}, V\right\rangle=-\int_{\Gamma} \sum_{l=1}^{k} q_{l} c_{j_{l}}^{2} f_{K_{j_{l}}}^{2}\left(x_{1}, \ldots, x_{d-1}\right) v_{d} \mathrm{~d} \sigma .
$$

Due to Proposition 2.6,

$$
\left.\sum_{l=1}^{k} q_{l} c_{j_{l}}^{2} f_{K_{j_{l}}}^{2}\right|_{\Gamma}
$$

is not everywhere zero on $\Gamma$. Thus, it is possible to choose $V=V_{j}$ for which $\left\langle\mathrm{d} G_{j}, V_{j}\right\rangle \neq 0$.

The conclusion follows by applying Theorem 2.4 with $F_{n}=1$ for $n \neq k$ and $F_{k}\left(y_{1}, \ldots, y_{k(k+1)}\right)=\sum_{i=1}^{k} q_{i} y_{k^{2}+i}$ and by taking $\hat{\mathcal{R}}_{j}=\left(\mathrm{Id}+t V_{j}\right)(\mathcal{R})$ for $t$ small enough.

\section{Applications to shape optimization And CONTRol theory}

\subsection{Stabilization of a damped membrane}

We consider here a stabilization problem in $\mathbb{R}^{2}$ and we are interested in proving the existence and uniqueness of solutions for a related shape optimization problem. More precisely, let us denote by $\Omega \subset \mathbb{R}^{2}$ a domain belonging to $D_{m}, m \in \mathbb{N} \cup\{\infty\}$. Assume that the Laplacian-Dirichlet eigenvalues of $\Omega$ are simple. 
We consider the problem of stabilizing a membrane fixed at the boundary $\partial \Omega$, thanks to a damping acting only on a subdomain $\omega$. Denote by $\chi_{\omega}$ the characteristic function of $\omega$. The displacement $v$ of the membrane, in presence of a viscous damping of the type $2 k \chi_{\omega}, k>0$, satisfies

$$
\begin{cases}\frac{\partial^{2} v}{\partial t^{2}}-\Delta v+2 k \chi_{\omega}(x) \frac{\partial v}{\partial t}=0 & (t, x) \in(0,+\infty) \times \Omega \\ v(t, x)=0 & x \in \partial \Omega, t>0 \\ v(0, x)=v_{0}(x) & x \in \Omega \\ \frac{\partial v}{\partial t}(0, x)=v_{1}(x) & x \in \Omega,\end{cases}
$$

where $v_{0} \in H_{0}^{1}(\Omega)$ and $v_{1} \in L^{2}(\Omega)$. This system is known to be exponentially stable if $\omega$ has positive measure and it is possible to define its exponential decay rate (which does not depend on the initial data). A natural question consists in looking for the largest decay rate once the area of $\omega$ is fixed. Such optimization problem is already quite difficult in the one-dimensional case (see e.g. [9]). For this reason Hébrard and Henrot in [11] introduce a simplified version of it by considering, instead of the decay rate, the quantity

$$
J_{N}(\omega):=\inf _{1 \leq n \leq N} \int_{\Omega} \chi_{\omega}(x)\left(\phi_{n}^{\Omega}(x)\right)^{2} \mathrm{~d} x
$$

where $N$ is a given positive integer and $\phi_{n}^{\Omega}$ denotes, as in the previous sections, the $n$th normalized LaplacianDirichlet eigenfunction.

Then, we are driven to study the following shape optimization problem

$$
\left\{\begin{array}{l}
\min J_{N}(\omega) \\
\omega \in \mathcal{L}_{\ell}
\end{array}\right.
$$

where $\mathcal{L}_{\ell}$ denotes the set of measurable subsets of $\Omega$ of measure $l$. It is convenient to identify subdomains of $\Omega$ with their characteristic functions, so that $\mathcal{L}_{\ell}$ is identified with

$$
\left\{a \in L^{\infty}(\Omega) \mid a(x)=0 \text { or } 1 \text { a.e. and } \int_{\Omega} a(x) \mathrm{d} x=\ell\right\}
$$

The one-dimensional problem is completely solved in [12]. In the same paper it is noticed that the proof of existence and uniqueness of the optimum for (3.3) can be easily adapted to the two-dimensional case under the generic hypothesis that the square of the Laplacian-Dirichlet eigenfunctions $\phi_{1}^{\Omega}, \ldots, \phi_{N}^{\Omega}$ are linearly independent (see Cor. 2.7). Indeed, first the authors prove the existence of an optimum $a^{*}$ in a relaxed class. In order to prove that such a maximum is a characteristic function, they study the optimality conditions satisfied by $a^{*}$, by considering perturbations of $a^{*}$ with support in $A_{\varepsilon}:=\left\{x \in \Omega \mid \varepsilon \leq a^{*}(x) \leq 1-\varepsilon\right\}$, with a small $\varepsilon>0$. They can prove in this way the existence of $N$ real numbers $\alpha_{1}, \ldots, \alpha_{N}$ such that $\alpha_{1}^{2}+\ldots+\alpha_{N}^{2} \neq 0$ and

$$
\sum_{k=1}^{N} \alpha_{k} \phi_{k}^{\Omega}(x)^{2}=\text { constant, for almost every } x \in A_{\varepsilon} .
$$

Then, because of the analyticity of the eigenfunctions and of the linear independence of their squares, $A_{\varepsilon}$ must have measure zero. 
Theorem 3.1. Generically with respect to $\Omega \in D_{m}$, the optimization problem (3.3) has a unique solution $\omega_{N}^{*}$.

\subsection{Controlled Schrödinger equation}

We apply in this section Corollary 2.8 in order to prove the generic approximate controllability of a bilinear Schrödinger equation of the type

$$
\begin{cases}i \frac{\partial \psi}{\partial t}(t, x)=(-\Delta+u(t) W(x)) \psi(t, x), & (t, x) \in(0,+\infty) \times \Omega \\ \psi(t, x)=0 & x \in \partial \Omega, t>0 \\ \psi(0, x)=\psi_{0}(x) & x \in \Omega,\end{cases}
$$

where $\Omega$ belongs to $\Sigma_{m}$ for some $m \in \mathbb{N} \cup\{\infty\}, W \in L^{\infty}(\Omega, \mathbb{R})$, the control $u$ belongs to $L^{\infty}([0,+\infty), U)$ for some fixed measurable subset $U$ of $\mathbb{R}$ with nonempty interior, and $\psi_{0} \in L^{2}(\Omega, \mathbb{C})$. System (3.4) admits always a mild solution $\psi \in \mathcal{C}\left([0,+\infty), L^{2}(\Omega, \mathbb{C})\right)$ in the sense of [5].

The control system (3.4) is said to be approximately controllable if for every $\psi_{0}, \psi_{1} \in L^{2}(\Omega, \mathbb{C})$ and every $\varepsilon>0$ there exist a control $u \in L^{\infty}([0,+\infty), U)$ and a positive time $T$ such that the solution $\psi$ of (3.4) satisfies $\left\|\psi(T, \cdot)-\psi_{1}\right\|_{L^{2}(\Omega)}<\varepsilon$.

It has been proved in [7] that (3.4) is approximately controllable if the Laplacian-Dirichlet operator on $\Omega$ has non-resonant spectrum and

$$
\int_{\Omega} W(x) \phi_{k}^{\Omega}(x) \phi_{k+1}^{\Omega}(x) \mathrm{d} x \neq 0 \quad \text { for every } k \in \mathbb{N} .
$$

Corollary 2.8 ensures that the Laplacian-Dirichlet spectrum is generically non-resonant. On the other hand, the unique continuation property implies that, for every $k \in \mathbb{N}$, the product $\phi_{k}^{\Omega} \phi_{k+1}^{\Omega}$ is a nonzero function on $\Omega$. Therefore, for every $\Omega$ with non-resonant spectrum, $\left\{W \in L^{\infty}(\Omega) \mid(3.5)\right.$ holds true $\}$ is residual in $L^{\infty}(\Omega)$. Moreover, due to the continuity of the eigenfunctions stated in Proposition 2.1, for every $k \in \mathbb{N}$ the map

$$
(\Omega, W) \mapsto \int_{\Omega} W(x) \phi_{k}^{\Omega}(x) \phi_{k+1}^{\Omega}(x) \mathrm{d} x
$$

is continuous with respect to the product topology of $\Sigma_{m} \times L^{\infty}\left(\mathbb{R}^{d}\right)$. As a consequence we obtain the following result.

Proposition 3.2. Generically with respect to $(\Omega, W) \in \Sigma_{m} \times L^{\infty}\left(\mathbb{R}^{d}\right)$, endowed with the product topology, system (3.4) is approximately controllable.

Acknowledgements. We would like to thank Yacine Chitour, Antoine Henrot and Enrique Zuazua for several fruitful discussions and advices.

\section{REFERENCES}

[1] A. Agrachev and M. Caponigro, Controllability on the group of diffeomorphisms. Preprint (2008).

[2] J.H. Albert, Genericity of simple eigenvalues for elliptic PDE's. Proc. Amer. Math. Soc. 48 (1975) $413-418$.

[3] W. Arendt and D. Daners, Uniform convergence for elliptic problems on varying domains. Math. Nachr. 280 (2007) $28-49$.

[4] V.I. Arnol'd, Modes and quasimodes. Funkcional. Anal. i Priložen. 6 (1972) 12-20.

[5] J.M. Ball, J.E. Marsden and M. Slemrod, Controllability for distributed bilinear systems. SIAM J. Control Optim. 20 (1982) $575-597$.

[6] K. Beauchard, Y. Chitour, D. Kateb and R. Long, Spectral controllability for 2D and 3D linear Schrödinger equations. J. Funct. Anal. 256 (2009) 3916-3976.

[7] T. Chambrion, P. Mason, M. Sigalotti and U. Boscain, Controllability of the discrete-spectrum Schrödinger equation driven by an external field. Ann. Inst. H. Poincaré Anal. Non Linéaire 26 (2009) 329-349.

[8] Y. Chitour, J.-M. Coron and M. Garavello, On conditions that prevent steady-state controllability of certain linear partial differential equations. Discrete Contin. Dyn. Syst. 14 (2006) 643-672. 
[9] S. Cox and E. Zuazua, The rate at which energy decays in a damped string. Comm. Partial Differential Equations 19 (1994) 213-243.

[10] Y.C. de Verdière, Sur une hypothèse de transversalité d'Arnol'd. Comment. Math. Helv. 63 (1988) 184-193.

[11] P. Hébrard and A. Henrot, Optimal shape and position of the actuators for the stabilization of a string. Systems Control Lett. 48 (2003) 199-209.

[12] P. Hébrard and A. Henrot, A spillover phenomenon in the optimal location of actuators. SIAM J. Control Optim. 44 (2005) 349-366 (electronic).

[13] A. Henrot and M. Pierre, Variation et optimisation de formes, Mathématiques et Applications 48. Springer-Verlag, Berlin (2005).

[14] L. Hillairet and C. Judge, Generic spectral simplicity of polygons. Proc. Amer. Math. Soc. 137 (2009) 2139-2145.

[15] T. Kato, Perturbation theory for linear operators, Die Grundlehren der mathematischen Wissenschaften 132. Springer-Verlag New York, Inc., New York (1966).

[16] J.-L. Lions and E. Zuazua, Approximate controllability of a hydro-elastic coupled system. ESAIM: COCV 1 (1995/1996) 1-15 (electronic).

[17] J.-L. Lions and E. Zuazua, A generic uniqueness result for the Stokes system and its control theoretical consequences, in Partial differential equations and applications, Lect. Notes Pure Appl. Math. 177, Dekker, New York (1996) 221-235.

[18] T.J. Mahar and B.E. Willner, Sturm-Liouville eigenvalue problems in which the squares of the eigenfunctions are linearly dependent. Comm. Pure Appl. Math. 33 (1980) 567-578.

[19] A.M. Micheletti, Metrica per famiglie di domini limitati e proprietà generiche degli autovalori. Ann. Scuola Norm. Sup. Pisa 26 (1972) 683-694.

[20] A.M. Micheletti, Perturbazione dello spettro dell'operatore di Laplace, in relazione ad una variazione del campo. Ann. Scuola Norm. Sup. Pisa. 26 (1972) 151-169.

[21] F. Murat and J. Simon, Étude de problèmes d'optimal design, Lecture Notes in Computer Sciences 41. Springer-Verlag, Berlin (1976).

[22] J.H. Ortega and E. Zuazua, Generic simplicity of the spectrum and stabilization for a plate equation. SIAM J. Control Optim. 39 (2000) 1585-1614 (electronic).

[23] J.H. Ortega and E. Zuazua, Generic simplicity of the eigenvalues of the Stokes system in two space dimensions. Adv. Differential Equations 6 (2001) 987-1023.

[24] J.H. Ortega and E. Zuazua, Addendum to: Generic simplicity of the spectrum and stabilization for a plate equation [SIAM J. Control Optim. 39 (2000) 1585-1614; mr1825594]. SIAM J. Control Optim. 42 (2003) 1905-1910 (electronic).

[25] J. Sokołowski and J.-P. Zolésio, Introduction to shape optimization: Shape sensitivity analysis, Springer Series in Computational Mathematics 16. Springer-Verlag, Berlin (1992).

[26] E.D. Sontag, Mathematical control theory: Deterministic finite-dimensional systems, Texts in Applied Mathematics 6. Springer-Verlag, New York (1990).

[27] M. Teytel, How rare are multiple eigenvalues? Comm. Pure Appl. Math. 52 (1999) 917-934.

[28] K. Uhlenbeck, Generic properties of eigenfunctions. Amer. J. Math. 98 (1976) 1059-1078.

[29] E. Zuazua, Switching controls. J. Eur. Math. Soc. (to appear). 